\title{
Preparation and Optical Limiting Properties of Multiwalled Carbon Nanotubes with $\pi$-Conjugated Metal-Free Phthalocyanine Moieties
}

\author{
Nan He, ${ }^{\dagger}$ Yu Chen, ${ }^{*, \dagger}$ Jinrui Bai, ${ }^{\dagger}$ Jun Wang, ${ }^{*,+}$ Werner J. Blau, ${ }^{*, \neq}$ and Jinhui Zhu ${ }^{\dagger}$ \\ Key Lab for Advanced Materials, Department of Chemistry, East China University of Science and Technology, \\ 130 Meilong Road, Shanghai 200237, China, and School of Physics and the Centre for Research on Adaptative \\ Nanostructures and Nanodevices (CRANN), Trinity College Dublin, Dublin 2, Ireland
}

Received: January 23, 2009; Revised Manuscript Received: May 11, 2009

\begin{abstract}
Phthalocyanines (Pcs) usually exhibit stronger optical limiting response that can be used to protect human eyes, optical elements, and sensors from intense laser pulses. New unsymmetrically substituted metal-free phthalocyanine-covalently functionalized multiwalled carbon nanotubes $\left(\mathrm{PcH}_{2}-\mathrm{MWNTs}\right)$, in which the wt \% of MWNTs in the resulting product was found to be $35 \%$, were synthesized. A considerably quenching of the fluorescence intensity was found in the photoluminescence spectrum of $\mathrm{PcH}_{2}-\mathrm{MWNTs}$. This observation suggests a quenching of the singlet excited $\mathrm{PcH}_{2}$ by the covalently linked MWNTs. This material exhibits strong scattering at higher intensities, which evidently comes from the MWNT counterpart. The nonlinear response of $t \mathrm{Bu}_{3} \mathrm{NH}_{2} \mathrm{PcH}_{2}$ is due to reverse saturable absorption (RSA), while that of $\mathrm{PcH}_{2}-\mathrm{MWNTs}$ is due to both RSA and nonlinear scattering, which could be two conflicted mechanisms for optical limiting, giving rise to suppression of the whole nonlinear response of $\mathrm{PcH}_{2}$-MWNTs.
\end{abstract}

\section{Introduction}

Aside from numerous civilian applications of lasers, they have evolved to numerous modern battlefield weapons. Some are designed to dazzle or permanently disable humans by blinding, while others are used to destroy optical sensors, missiles, and other targets. ${ }^{1}$ The need for passive laser protectors to protect human eyes and all optical sensors from intense laser beams is not limited to the military but is rather a growing societal problem that can only escalate. ${ }^{2}$ In the past decade, significant research effort has been invested into optical limiting materials and processes in an attempt to achieve some measure of protection from such laser beams. ${ }^{3-8}$ Preparation of the required nonlinear optical active materials for practical optical limiters, however, still presents a significant chemical challenge.

Third-order optical nonlinearities of phthalocyanines (Pcs) are of great interest due to their highly delocalized aromatic 18 $\pi$-electron systems giving rise to strong reverse saturable absorption (RSA) properties, exceptional stability, processability features, and their architectural flexibility which allows tailoring of their physical, optoelectronic, and chemcial parameters in a broad rang as well. ${ }^{9}$ Similar to $\mathrm{C}_{60}$ and its organic and polymeric derivatives ${ }^{3 \mathrm{~b}} \mathrm{Pcs}$ are materials that optically limit nanosecond light pulses in a fairly wide range of the UV/vis absorption spectrum via excited state absorption processes. ${ }^{6,79}$ In our previous work, we utlized the chemical reactivity of the $\mathrm{M}-\mathrm{Cl}$ $\left[\mathrm{M}=\mathrm{Ga}^{3+}, \mathrm{In}^{3+}\right]$ and $\mathrm{M}=\mathrm{O}\left[\mathrm{M}=\mathrm{Ti}^{4+}\right.$, etc. $]$ bonds to synthesize a series of highly soluble axially substituted and bridged Pc-based organic compounds, all of which exhibit very good optical limiting response. ${ }^{10}$

To achieve simultaneous protection against both pulsed and continuous wave $(\mathrm{CW})$ or quasi-CW lasers, broadband optical limiting chromophores needed to be designed for multimecha-

\footnotetext{
* To whom correspondence should be addressed. E-mail: chentangyu@ yahoo.com (Y.C.); jwangsci@gmail.com (J.W.); wblau@tcd.ie (W.J.B.)

${ }^{\dagger}$ East China University of Science and Technology.

* Trinity College Dublin.
}

nistic function, ${ }^{2}$ which encouraged us to design and prepare a new phthalocyanine covalently functionalized carbon nanotubes (CNTs) by combination of the advantages of phthalocyanines and the outstanding broadband optical limiting response of CNTs. ${ }^{11}$ The optical limiting responses of carbon nanotube suspensions are shown to be dominated by nonlinear scattering as a result of thermally induced solvent-bubble formation and sublimation of the nanotubes, while the solubilized carbon nanotubes optically limit through nonlinear absorption mechanism and exhibit significant solution-concentration-dependent optical limiting responses. For the polymer/carbon nanotube composites, the optical limiting mechanism is quite complicated. Besides nonlinear scattering contribution to the optical limiting, there may also be other contributions, for example, nonlinear absorption, nonlinear refraction, electronic absorption, and others to the optical limiting. ${ }^{11}$

The chemistry of CNTs is a current subject of intense research which produces continuous advances and novel functional materials. ${ }^{12}$ The possibe organic reactions of CNTs usually include (1) the addition of 1,3-dipoles and dienes, (2) the addition of nucleophiles, and the addition of radicals. de la Torre et al. ${ }^{13}$ were the first to describe the synthesis of single-walled nanotubes covalently attached to zinc phthalocyanine (PcZn) molecules through amide bonds, which were scarcely soluble in some common organic solvents, and simply characterized by FTIR, UV/vis, and TEM. This kind of multichromophoric system based on Pcs constitutes an appealing architecture to exploit both the electronic and photonic interactions between individual subunits. Very recently Torres and co-workers ${ }^{14}$ reported on the preparation of new dispersible $\mathrm{ZnPc}$ singlewalled carbon nanotube (SWNT) hybrids through the Prato reaction of HiPco SWNT, $N$-octylglycine, and adequately functionalized $\mathrm{ZnPc}$ molecules, bearing six solublizing groups and an aldehyde. The occurrence of electron transfer from photoexcited Pcs to the nanotube framework in these ZnPcSWNT ensembles is observed in transient absorption experiments. Basically a perturbation of the electronic distribution in 


\section{SCHEME 1: Preparation of $\mathbf{P c H}_{2}$ Covalently Functionalized MWNTs}
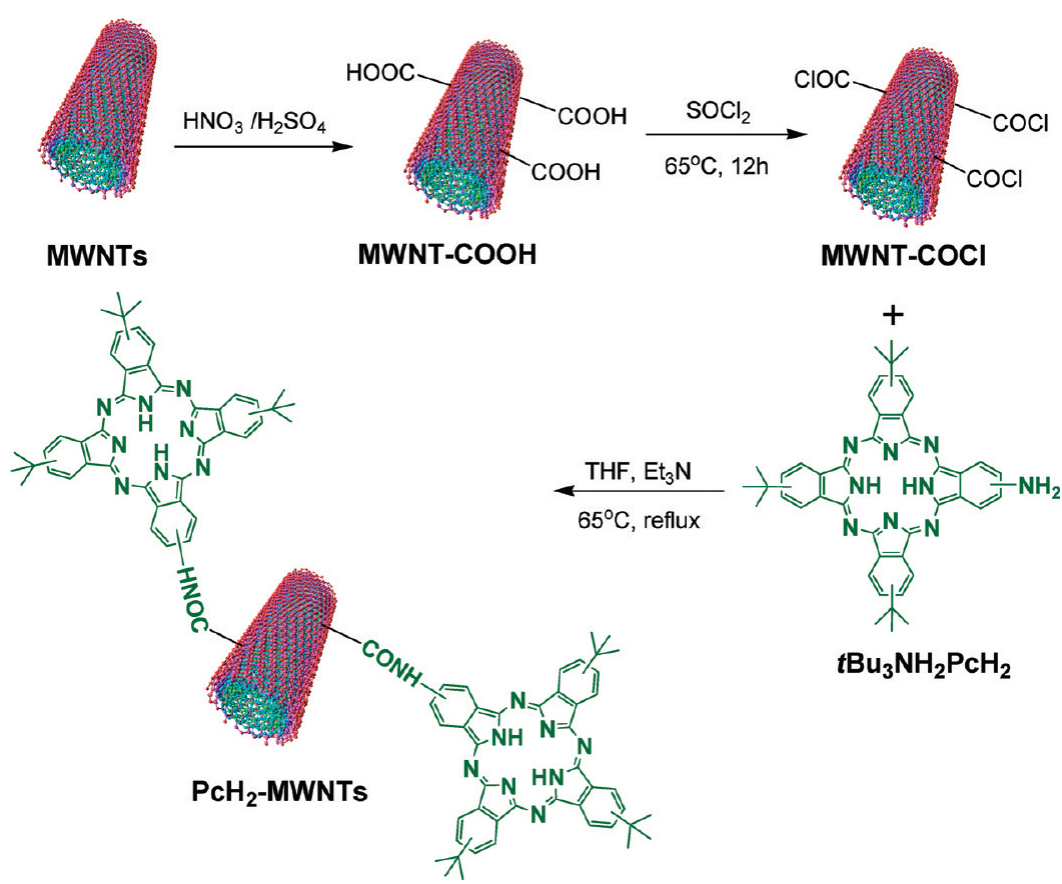

the material as a response to the electric field of the incident (low intensity) light is reason for normal linear polarization to occur. At high intensities, the electronic distribution no longer follows the applied field, resulting in both second- and thirdorder nonlinearities. ${ }^{6}$ In this contribution, an unsymmetrically substituted metal-free aminophthalocyanine $\left[t \mathrm{Bu}_{3} \mathrm{NH}_{2} \mathrm{PcH}_{2}\right]^{15}$ was directly reacted with the multiwalled carbon nanotubes (MWNTs) with surface-bonded acryl chloride moieties to give $\mathrm{PcH}_{2}$-MWNTs nanocomposites, as shown in Scheme 1. MWNTs are the ensemble of many concentric graphene cylinders, and their diameter ranges typically between 10 and $100 \mathrm{~nm}$. It is thus generally believed that the interactions between the shells as well as the large diameter to length ratio may alter the electronic structure of these MWNTs, which may be significantly different from that of SWNTs. The covalent attachment of $\mathrm{PcH}_{2}$ moieties onto the surfaces of the MWNTs has been confirmed by a variety of physical and spectral techniques. This material exhibits strong scattering at higher intensities, which evidently comes from the MWNT counterpart. Combination of $\mathrm{PcH}_{2}$ and MWNTs effectively extends the optical limiting response wavelength from the visible to the near-infrared.

\section{Experimental Section}

General Materials and Methods. All chemicals were purchased from Aldrich and used without further purification. Organic solvents were purified, dried, and distilled under dry nitrogen. The MWNT sample was purchased from Tsinghua Nano-Powder Engineering Centre (Beijing, China) with a diameter of $10-20 \mathrm{~nm}$, a length of $5-15 \mu \mathrm{m}$, and a $>95 \%$ purity. The metal-free tri-tert-butylaminophthalocyanine, $t \mathrm{Bu}_{3} \mathrm{NH}_{2} \mathrm{PcH}_{2}$, was synthesized according to the literature as reported. ${ }^{15}$ The operations for synthesis prior to the termination reaction were carried out under purified argon.

Fourier transform infrared (FTIR) spectra were recorded on a Nicolet Nagma-IR 550 spectrophotometer using KBr pellets. Raman spectra were taken at room temperature with a MicroRaman System RM3000 spectrometer and an argon ion laser operating at a wavelength of $514.5 \mathrm{~nm}$ as the excitation source.
The UV/vis spectral measurements were carried out with a Shimadzu UV-2450 spectrophotometer. Thermal properties of the samples were measured using a Perkin-Elmer Pyris 1 thermogravimetric analyzer in flowing $\left(100 \mathrm{~mL} \cdot \mathrm{min}^{-1}\right)$ nitrogen atmosphere. Steady-state fluorescence spectra were measured on a Shimadzu RF-5300 PC spectrofluorophotometer equipped with a photomultiplier tube having high sensitivity in the 700-800 $\mathrm{nm}$ region. The sample for the fluorescence measurement was dissolved in the dry toluene, filtered, transferred to a long quartz cell, and then capped and bubbled with high pure argon (without $\mathrm{O}_{2}$ and moisture) for at least $15 \mathrm{~min}$ before measurement. Atomic force microscopy (AFM) images were obtained using tapping mode on a Nanoscope IV instrument from Digital Instruments equipped with a silicon cantilever 125 $\mu \mathrm{m}$ and E-type vertical engage piezoelectric scanner. The AFM samples were prepared by spin-coating the samples on the freshly cleaved mica and then dried naturally at room temperature for at least $24 \mathrm{~h}$. Transmission electron microscopy (TEM) images were recorded on a JEM-2100S TEM system operated at $100 \mathrm{kV}$. Scanning electron microscopy (SEM) images were recorded on a S-2150 Hitachi microscope. Before measurement, the samples were precoated with a homogeneous gold layer by sputtering technology.

All the Z-scan experiments were performed using $6 \mathrm{~ns}$ pulses from a Q-switched Nd:YAG laser. The beam was spatially filtered to remove higher-order modes and tightly focused with a $9 \mathrm{~cm}$ focal length lens. The laser was operated at its second harmonic, $532 \mathrm{~nm}$, with a pulse repetition rate of $10 \mathrm{~Hz}$. Simultaneously, a focusing lens setup was arranged at $\sim 30^{\circ}$ to the direct incident beam to monitor the scattered light from dispersions. All samples were tested in $0.1 \mathrm{~cm}$ quartz cells.

Preparation of MWNT-COCl. A total of $5.0 \mathrm{~g}$ of crude MWNTs was marinated in $38 \% \mathrm{HCl}$ for 1 day, filtered, and washed with deionized water until neutral. A mixture of MWNTs, $\mathrm{HNO}_{3}(30 \mathrm{~mL}, 60 \%)$, and $\mathrm{H}_{2} \mathrm{SO}_{4}(90 \mathrm{~mL}, 98 \%$ ) was sonicated at $40{ }^{\circ} \mathrm{C}$ for $0.5 \mathrm{~h}$ first and then was refluxed for $2 \mathrm{~h} .{ }^{16}$ After termination of reaction, it was allowed to cool to room tempeture. The mixture was diluted with a large amount of 
deionized water, followed by a vacuum-filtering through a Nylon film $(\phi 0.45 \mu \mathrm{m})$. The obtained solid was washed with water until the aqueous layer reached neutral, and then it was vacuumdried at $60{ }^{\circ} \mathrm{C}$ for $3 \mathrm{~h}$. The COOH-containing MWNTs $(500$ $\mathrm{mg}$ ) were reacted with a large excess of $\mathrm{SOCl}_{2}$ containing a catalytic amount of dimethylformamide (DMF) under reflux for 24 h. ${ }^{17}$ After centrifugation, the remaining solid was washed with anhydrous tetrahydrofuran (THF) to remove the residual thionyl chloride.

Preparation of $\mathbf{P c H}_{2}$-MWNTs. The surface-bound acrychloride groups in $\mathrm{MWNT}-\mathrm{COCl}$ were used to react with the $\mathrm{NH}_{2}$ group of $t \mathrm{Bu}_{3} \mathrm{NH}_{2} \mathrm{PcH}_{2}$ to give $\mathrm{PcH}_{2}$-MWNTs nanocomposite material. Typically, to a suspension of MWNT-COCl (500 $\mathrm{mg})$ in anhydrous THF $(70 \mathrm{~mL})$ was added to $t \mathrm{Bu}_{3} \mathrm{NH}_{2} \mathrm{PcH}_{2}$ $(55 \mathrm{mg})$ and $\mathrm{Et}_{3} \mathrm{~N}(17 \mathrm{~mL})$ under purified argon. The reaction mixture was sonicated $1 \mathrm{~h}$ at $40^{\circ} \mathrm{C}$ first and then refluxed at 65 ${ }^{\circ} \mathrm{C}$ for 4 days. It should be noted that light must be strictly excluded during this step in order to avoid possible photodegradation of phthalocyanine. After cooling to room temperature and removing the solvents by centrifugation, a large amount of water was added to the solid residue to remove the triethylammonium salts formed during the reaction. A total of $200 \mathrm{~mL}$ of THF was then added to the above reaction mixture under stirring. The obtained blue-green solution was vacuum-filtered through a single layer Nylon film $(\phi 0.45 \mu \mathrm{m})$ until the filtrate became colorless. The black solid on the Nylon film was collected and then was well-dispersed in THF under the ultrasonic irradiation $(40 \mathrm{kHz})$, followed by filtering through a five-layer filter paper (this procedure was repeated at least five times until the filtration became colorless). The crude product collected after evaporation of the solvent was subjected to recrystallization from a mixture of $\mathrm{CH}_{2} \mathrm{Cl}_{2} / \mathrm{MeOH}$ (v/v 4:3) by slowly evaporating the more volatile dichloromethane in a rotary evaporator at $40-60{ }^{\circ} \mathrm{C}$ under slightly reduced pressure. The resultant product, a bluish-black solid, was collected by filtration, washed twice with methanol, and dried at $60{ }^{\circ} \mathrm{C}$ in vacuo for 10 h. Yield: $87.6 \mathrm{mg}$.

\section{Results and Discussion}

A mixed condensation of the 4-tert-butylphthalonitrile (3 equiv) and 4-nitrophthalonitrile (1 equiv) at $180{ }^{\circ} \mathrm{C}$ gives a mixture of mono- through to tetranitrophthalocyanine derivatives which were further treated with $\mathrm{Na}_{2} \mathrm{~S} \cdot 9 \mathrm{H}_{2} \mathrm{O}$ in DMF at $65^{\circ} \mathrm{C}$ to yield a mixture of mono- and polyaminophthalocyanines. ${ }^{15}$ In contrast to the nitrophthalocyanines, the more polar amino derivatives could be easily separated on a silica gel column [toluene-ethylacetate (v/v 5:1) as eluting solvents], and gave the pure tri-tert-butylaminophthalocyanine $t \mathrm{Bu}_{3} \mathrm{NH}_{2} \mathrm{PcH}_{2}$. The proton signal for the $\mathrm{NH}_{2}$ group in the Pc structure appears at $\delta=4.2 \mathrm{ppm}$. The condensation of $t \mathrm{Bu}_{3} \mathrm{NH}_{2} \mathrm{PcH}_{2}$ and MWNT$\mathrm{COCl}$ gives metal-free phthalocyanine covalently functionalized MWNTs. In the ${ }^{1} \mathrm{H}$ NMR spectrum, the $\mathrm{NH}_{2}$ signal disappears, followed by the appearance of a new proton signal at $\delta=8.2$ ppm which was assigned to the formation of an amido bond between Pc and MWNTs. As expected, the aromatic protons of the macrocycles are deshielded and thus their signals are shifted downfield due to the covalent attachment of Pc moieties onto the surfaces of MWNTs. Like [60]fullerene, MWNTs can also act as an electron-acceptor. Although organic groups or species can be theoretically of addition to both the internal and external surfaces of CNTs, very reactive reagents are required. ${ }^{18}$

Raman spectroscopy provides essential and useful information for the covalent attachment of the organic and/or polymeric functional groups onto the surface of CNTs. There are, basically,

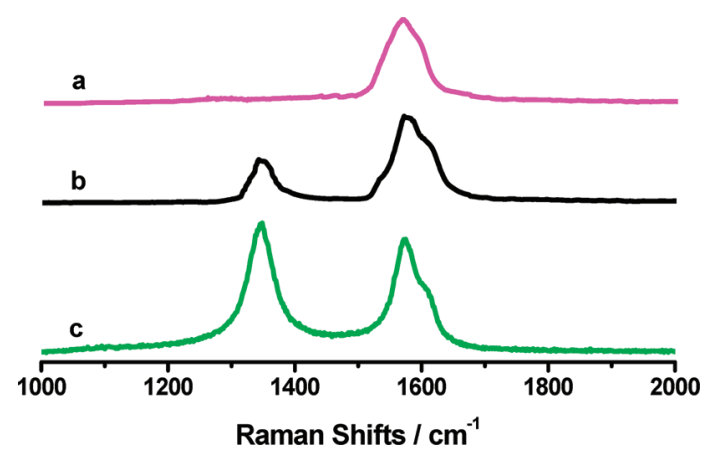

Figure 1. Raman spectra of (a) pristine MWNTs, (b) MWNT-COOH, and (c) $\mathrm{PcH}_{2}$-MWNTs.

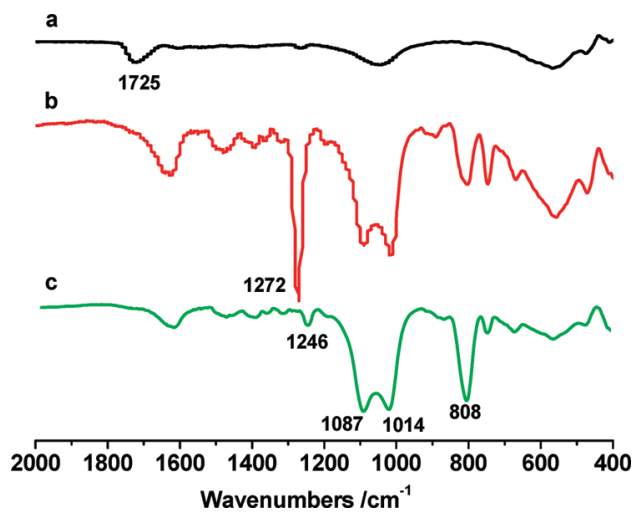

Figure 2. IR spectra of (a) MWNT-COOH, (b) $t \mathrm{Bu}_{3} \mathrm{NH}_{2} \mathrm{PcH}_{2}$, and (c) $\mathrm{PcH}_{2}$-MWNTs.

two prominent bands at about 1330-1380 (D-band) and $1400-1600$ (G-band) $\mathrm{cm}^{-1}$ in the Raman spectrum of CNTs. ${ }^{14,19}$ The former indicates, as usual for CNT structures, the density of defects and has been used to monitor the process of covalent functionalization which transforms $\mathrm{sp}^{2}$ to $\mathrm{sp}^{3}$ sites, while the latter could be utlized to estimate the level and distribution of modification. As shown in Figure 1 (514.5 nm excitation), the pristine MWNTs exhibit a band at $1575 \mathrm{~cm}^{-1}$ (G-band). After acid treatment, the observed D- and G-bands appear at 1342 and $1573 \mathrm{~cm}^{-1}$, respectively, being attributed to the defects and disorder-induced modes and in-plane $E_{2 \mathrm{~g}}$ zone-center mode. ${ }^{17 \mathrm{a}}$ The $\mathrm{D}^{\prime}$-band, which is directly affected by the disorder in carbon nanotubes, is located at $1610 \mathrm{~cm}^{-1}$. In contrast to the case of MWNT-COOH, the D- and G-bands of $\mathrm{PcH}_{2}-\mathrm{MWNT}$, which appear at 1347 and $1578 \mathrm{~cm}^{-1}$, are found to be slightly shifted to the high wavenumbers. The $\mathrm{D}$ - to G-band intensity ratios $\left(I_{\mathrm{D}} / I_{\mathrm{G}}\right)$ for MWNT-COOH and $\mathrm{PcH}_{2}-\mathrm{MWNT}$ are about 0.3 and 1.3 , respectively. The latter is about 4.3 times that of the former, suggesting a significant modification after the addition of phthalocyanine via a covalent bond. An analogous phenomenon was also observed by other groups. ${ }^{20,21}$

From the IR spectrum of MWNT-COOH (Figure 2a), it can be seen that the characteristic vibration for the carboxylic acid groups attached onto the surfaces of MWNTs appears at 1725 $\mathrm{cm}^{-1}\left(v_{\mathrm{C}=\mathrm{O}}\right) \cdot t \mathrm{Bu}_{3} \mathrm{NH}_{2} \mathrm{PcH}_{2}$ exhibits a typical IR spectrum of metal-free phthalocyanine, in which the bands at 1630 and 1272 $\mathrm{cm}^{-1}$ are attributed to the $\mathrm{C}=\mathrm{C}$ stretch mode of the macrocycle and to the $\mathrm{C}-\mathrm{N}$ stretch mode of the primary aromatic amine, respectively. Comparing the IR spectra of MWNT-COOH, $t \mathrm{Bu}_{3} \mathrm{NH}_{2} \mathrm{PcH}_{2}$, and $\mathrm{PcH}_{2}-\mathrm{MWNT}$ reveals that the absorption bands at 1725 and $1272 \mathrm{~cm}^{-1}$ disappear in the resultant material due to the covalent attachment of Pc moieties onto MWNTs, followed by the appearance of a weak new band at $1246 \mathrm{~cm}^{-1}$ and by the enhancement of intensities of some absorption bands 


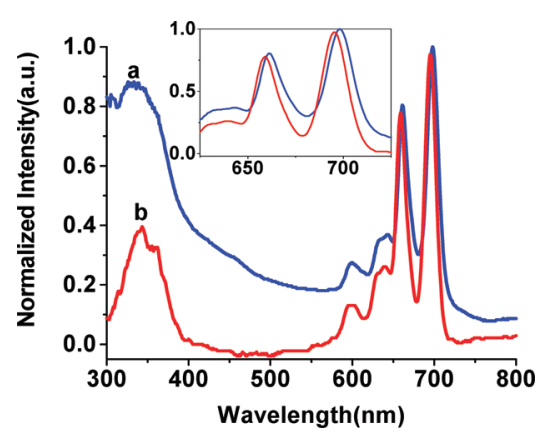

Figure 3. UV/vis absorption spectra of (a) $\mathrm{PcH}_{2}-\mathrm{MWNT}$ and (b) $t \mathrm{Bu}_{3} \mathrm{NH}_{2} \mathrm{PcH}_{2}$ in toluene.

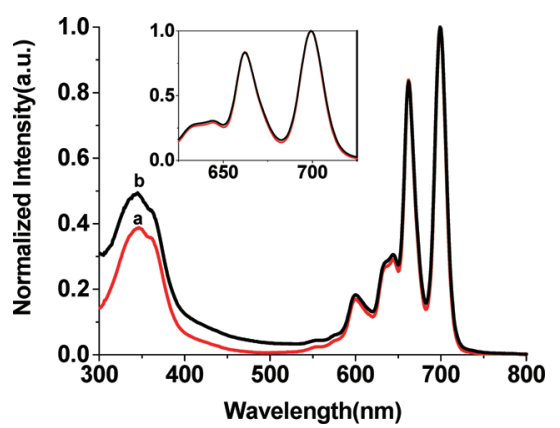

Figure 4. UV/vis absorption spectra of (a) $t \mathrm{Bu}_{3} \mathrm{NH}_{2} \mathrm{PcH}_{2}$ and (b) a mixture of $\mathrm{PcH}_{2}$ and MWNTs in toluene.

at, for example, 1087 and $808 \mathrm{~cm}^{-1}$. The characteristic vibration for the amido linkage between Pc and MWNTs was possiblly hidden in the IR absorption bands of phthalocyanine.

The electronic absorption spectra of phthalocyanines are characterized by an intense Q-band in the red end of the visible spectrum of light between $600-700 \mathrm{~nm}$, with a molar absorption often exceeding $10^{5} \mathrm{~cm}^{2} / \mathrm{mol}$, and a B-band at $300-400 \mathrm{~nm}$ in the blue end of the visible spectrum. In the case of metal-free phthalocyanines, all states are nondegenerated, due to the reduced $D_{2 \mathrm{~h}}$ molecular symmetry. The Q-band transition is polarized in either the $x$ or $y$ direction and is therefore split into two bands. ${ }^{9 b}$ From Figure 3, one can clearly see that, in contrast to the case of $t \mathrm{Bu}_{3} \mathrm{NH}_{2} \mathrm{PcH}_{2}$, the peak maxima of the Q- and B-bands of $\mathrm{PcH}_{2}-\mathrm{MWNTs}$ move to the red by $\Delta \lambda=3 \mathrm{~nm}$ and to the blue by $\Delta \lambda=2 \mathrm{~nm}$, respectively, as a consequence of the electronic interactions with MWNTs ( $\mathrm{Pc}$ is usually regarded as the electron donor, while MWNTs as electron acceptor). The relative intensity and the peak shape of the B-band are found to considerably increase and to substantially broaden, respectively. Even so, the spectral window between the Q- and B-bands is still clearly evident, suggesting that the $\mathrm{PcH}_{2}-\mathrm{MWNT}$ composite material is still suitable for optical limiting of 532 nm light. The linear absorption coefficients $\left(\alpha_{0}\right)$ of $\mathrm{PcH}_{2}-$ MWNTs in toluene is slightly smaller than that of $t \mathrm{Bu}_{3} \mathrm{NH}_{2} \mathrm{PcH}_{2}$ in the same solution $\left(0.95\right.$ against $1.18 \mathrm{~cm}^{-1}$ for the latter). Basically, the electronic absorption spectrum of MWNTs suspended in organic solvent is very weak. The UV/vis absorption spectrum of the blend of MWNTs and phthalocyanine in toluene only exhibits the characteristic absorption of the Pc macromolecules (Figure 4). All these findings are indicative of the formation of a covalent bond between Pc and MWNTs.

The photoexcited states of $t \mathrm{Bu}_{3} \mathrm{NH}_{2} \mathrm{PcH}_{2}$ and $\mathrm{PcH}_{2}$-MWNTs were investigated by steady-state fluorescence measurements (Figure 5). When the sample was excited with $300 \mathrm{~nm}$ light, the photoluminescence spectra of both materials were almost similar, but the emission maximum of $\mathrm{PcH}_{2}$-MWNTs was found

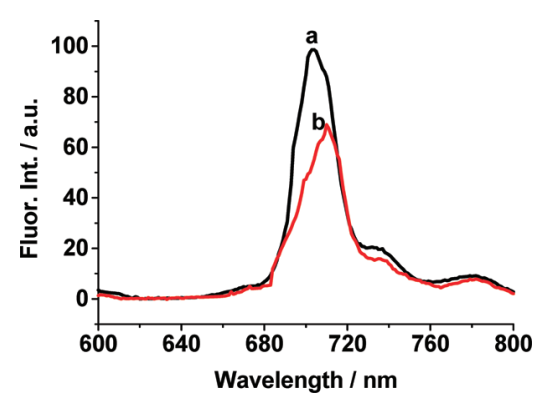

Figure 5. Steady-state fluorescence spectra of (a) $t \mathrm{Bu}_{3} \mathrm{NH}_{2} \mathrm{PcH}_{2}$ and (b) $\mathrm{PcH}_{2}$-MWNTs in toluene. $\lambda_{\mathrm{ex}}=300 \mathrm{~nm}$.

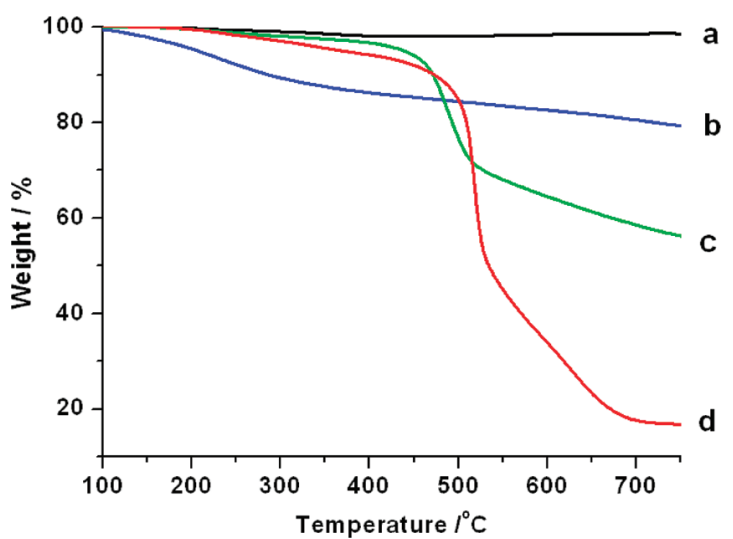

Figure 6. TGA profiles of (a) pristine MWNTs, (b) MWNT-COOH, (c) $\mathrm{PcH}_{2}-\mathrm{MWNTs}$, and (d) $t \mathrm{Bu}_{3} \mathrm{NH}_{2} \mathrm{PcH}_{2}$. Experiments were carried out in highly purified nitrogen atmosphere.

to be shifted to the red by $\Delta \lambda=7 \mathrm{~nm}$ when compared to that of $t \mathrm{Bu}_{3} \mathrm{NH}_{2} \mathrm{PcH}_{2}$. Considering such a fact that MWNT suspensions did not display any fluorescence signal, it is thus reasonable that the fluorescence of $\mathrm{PcH}_{2}-\mathrm{MWNTs}$ was from the $\mathrm{PcH}_{2}$ moieties. A considerable quenching of the fluorescence intensity was found in the photoluminescence spectrum of $\mathrm{PcH}_{2}$ MWNTs. This observation suggests a quenching of the singlet excited $\mathrm{PcH}_{2}$ by the covalently linked MWNTs. Similar observation has also been reported by other groups. ${ }^{22}$

Basically the actual amount of $\mathrm{C}_{60}$ incorporated in the polymer can be determined by thermogravimetry (TG). ${ }^{23}$ Based on this conclusion, the amount of MWNTs in the $\mathrm{PcH}_{2}$-MWNTs seems to be able to be determined using a similar method mentioned above. From Figure 6, it can be seen that the onset temperature for the thermal bond cleavage of pristine MWNTs is about 110 ${ }^{\circ} \mathrm{C}$ and the weight loss between 110 and $230{ }^{\circ} \mathrm{C}$ is about $4 \%$, which is due to the possible organic and inorganic impurities (including possible organic solvents) trapped in MWNTs. The decomposited MWNT residues seems to be very stable over a wide termperature range of $240-750^{\circ} \mathrm{C}$. In contrast, the acidfunctionalized MWNT-COOH is less thermally stable perhaps due to the defects on the nanotube surface caused by the acid etching. ${ }^{24}$ The TGA curve of MWNT-COOH exhibited a rapid weight loss of $15 \%$ between 100 and $400{ }^{\circ} \mathrm{C}$, followed by a very slow weight loss of $5 \%$ up to $750{ }^{\circ} \mathrm{C}$ at which the amount of the MWNT residue is about $80 \%$. By comparing the amount of the residues these two samples left at $750{ }^{\circ} \mathrm{C}$, the wt $\%$ of oxygen-containing groups at CNT defect sites was roughly estimated to be about $16 \%$. The TGA curve of $t \mathrm{Bu}_{3} \mathrm{NH}_{2} \mathrm{PcH}_{2}$ shows two plateaus. The residue of $18 \%$ was obtained at 750 ${ }^{\circ} \mathrm{C}$. By assuming that at $750{ }^{\circ} \mathrm{C}$ the $\mathrm{Pc}$ residues remaining in $\mathrm{PcH}_{2}$-MWNTs have the same wt $\%$ as that of the $t \mathrm{Bu}_{3} \mathrm{NH}_{2} \mathrm{PcH}_{2}$ complex, and the wt \% of oxygen-containing groups at CNT defect sites is close to zero at this temperature, the wt $\%$ of 

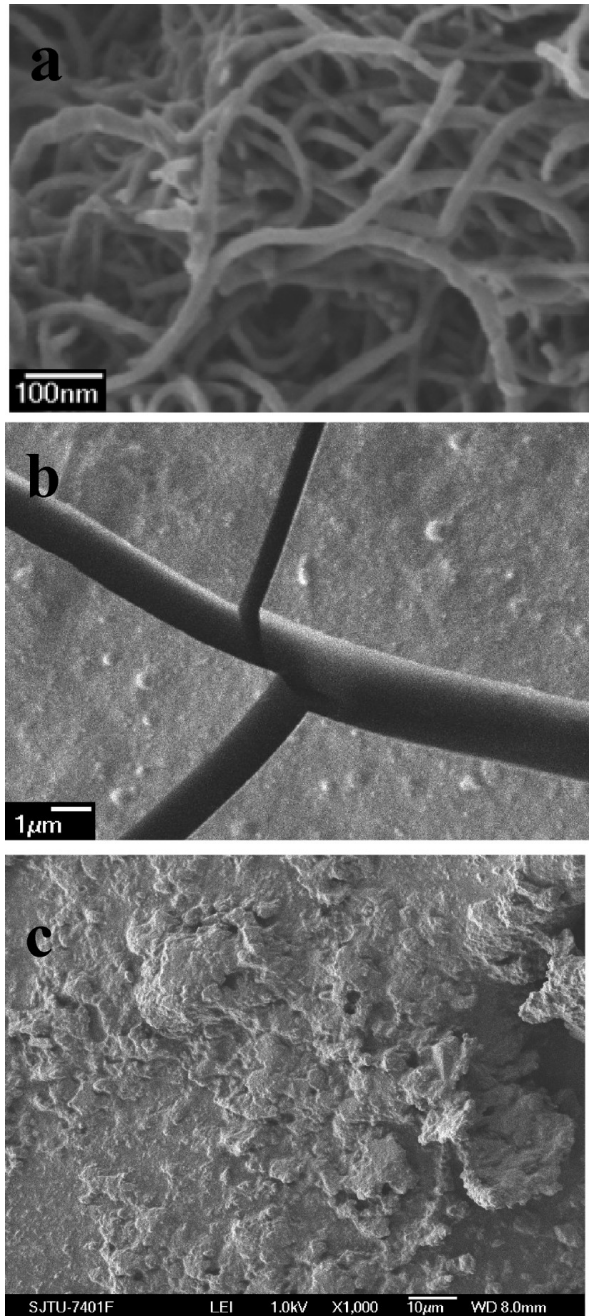

Figure 7. SEM images of (a) MWNT-COOH, (b) $\mathrm{PcH}_{2}-\mathrm{MWNT}$, and (c) $t \mathrm{Bu}_{3} \mathrm{NH}_{2} \mathrm{PcH}_{2}$.

MWNTs in the resulting product may be roughly calculated, and it was found to be $35 \%$ from which one can understand why the resulting material is soluble in some common organic solvents.

The morphologies of the samples MWNT-COOH, $t \mathrm{Bu}_{3} \mathrm{NH}_{2-}$ $\mathrm{PcH}_{2}$, and $\mathrm{PcH}_{2}-\mathrm{MWNT}$ were observed with SEM (Figure 7) and AFM (Figure 8). From Figure 7, it can be seen that, after the ultrasonic and strong acid treatment, the acidified MWNTs, that is, MWNT-COOH, with the average diameter of $20 \mathrm{~nm}$, still keep the intrinsic quality of nanotube structures. As expected, metal-free phthalocyanine, $t \mathrm{Bu}_{3} \mathrm{NH}_{2} \mathrm{PcH}_{2}$, exhibits typical aggregated features, which is different from that of the acidified MWNTs. Like many of the organic dyes, phthalocyanine without axial-substituents tends to aggregate at high concentration, especially in the solid state. These aggregates are usually depicted as a coplanar association of rings progressing from monomer to dimer and higher order complexes, and are driven by enhanced van der Waals attractive forces between phthalocyanine rings. ${ }^{25}$ After grafting of $\mathrm{PcH}_{2}$ onto the surface of MWNTs, the functionalized tubes appear to form dendritical structures from which one can easily find that the largest diameter of MWNTs covalently coated with $\mathrm{PcH}_{2}$ moieties is about $2 \mu \mathrm{m}$. As shown in Figure 8, the AFM image of $t \mathrm{Bu}_{3} \mathrm{NH}_{2} \mathrm{PcH}_{2}$ consists of many egg-shaped species with a diameter of 50-170 $\mathrm{nm}$. This result is suggestive of aggregation of $\mathrm{PcH}_{2}$ molecules in the solid state due to a strong $\pi-\pi$ stacking effect between Pc macrocycles. In contrast to the
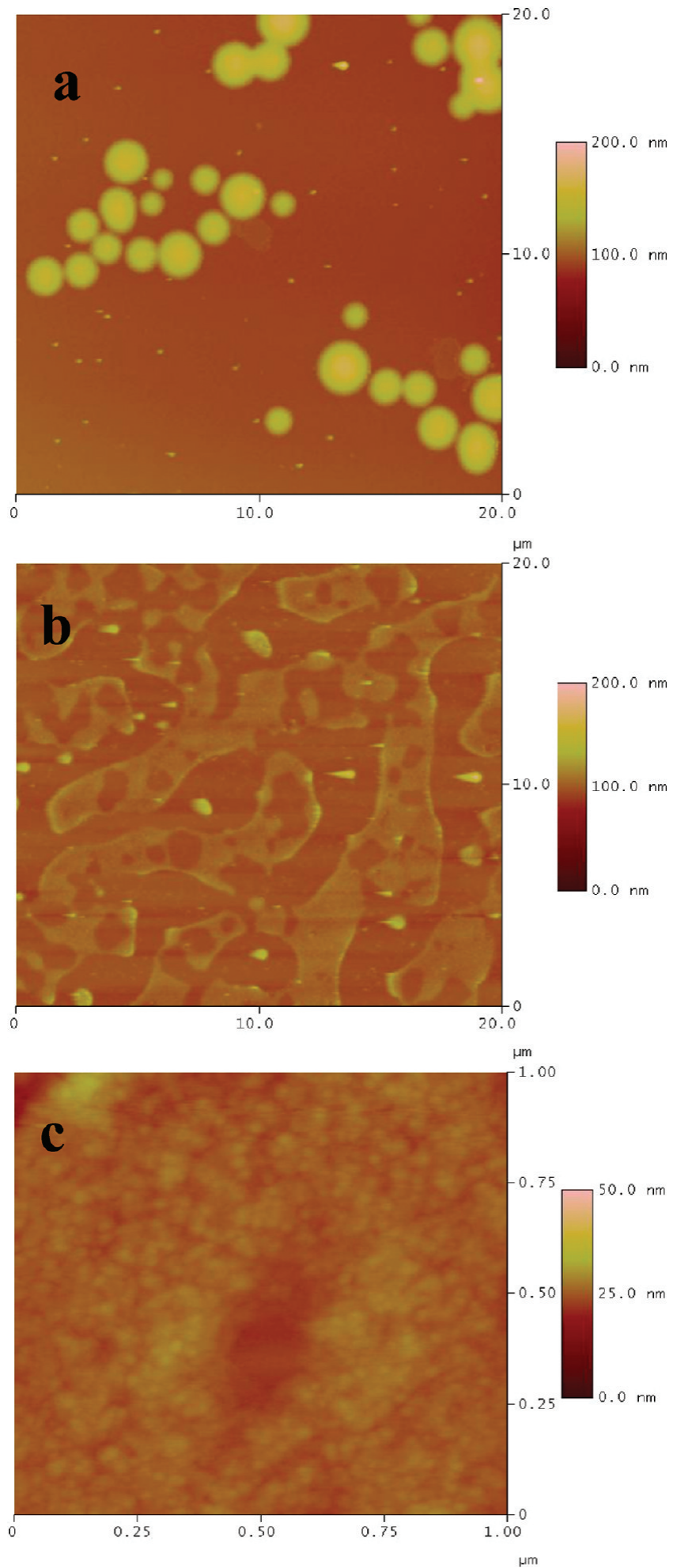

Figure 8. AFM images of (a) $t \mathrm{Bu}_{3} \mathrm{NH}_{2} \mathrm{PcH}_{2}$, (b) $\mathrm{PcH}_{2}$-MWNTs (general view, low magnification image), and (c) $\mathrm{PcH}_{2}-\mathrm{MWNT}$ (high magnification image).

$t \mathrm{Bu}_{3} \mathrm{NH}_{2} \mathrm{PcH}_{2}$ complex, the AFM images of $\mathrm{PcH}_{2}-\mathrm{MWNT}$ (Figure $8 \mathrm{~b}$ and $\mathrm{c}$ ) are completely different from that of the former. From the airscape, the general view of $\mathrm{PcH}_{2}-\mathrm{MWNT}$ looks like slices at low magnification. In the case of high magnification, one can observe the agglomeration pattern of phthalocyanine moieties formed onto the outer layer of MWNTs. As expected, the use of AFM did not allow us to see the fine structure of the MWNTs. 

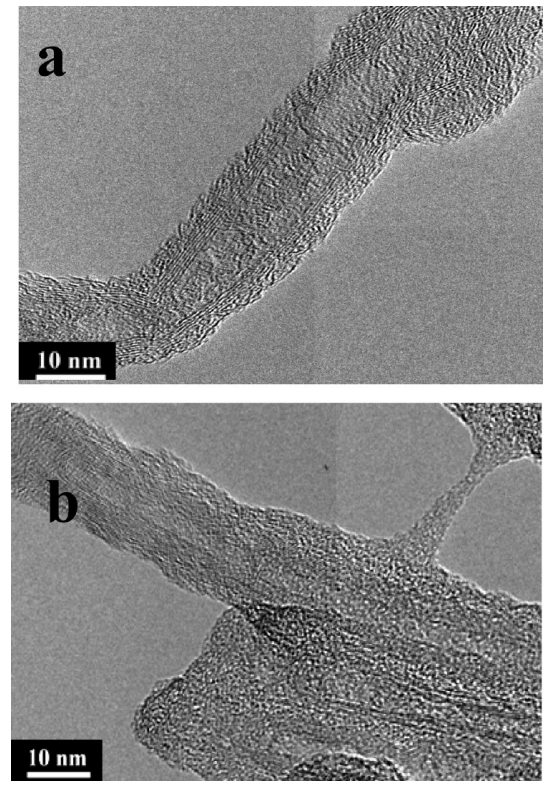

Figure 9. TEM images of (a) MWNT-COOH and (b) $\mathrm{PcH}_{2}-\mathrm{MWNT}$.

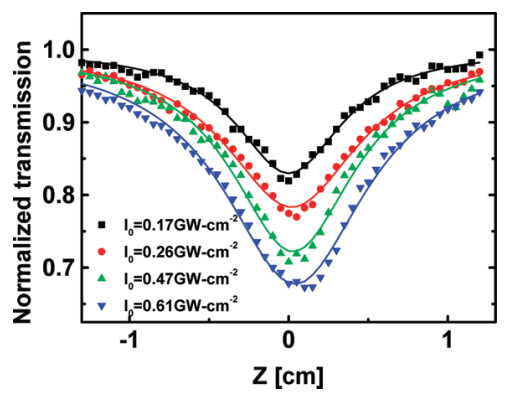

Figure 10. Typical open aperture $\mathrm{Z}$-scan curves of $\mathrm{PcH}_{2}$-MWNTs with the concentration of $0.5 \mathrm{mg} / \mathrm{mL}$. Solid lines are the numerical fittings.

The fine nanostructures of MWNT-COOH and $\mathrm{PcH}_{2}-\mathrm{MWNTs}$ were investigated by high-resolution TEM (HRTEM) which is a powerful tool for characterizing nanomaterials including inorganic nanoparticles, CNTs, and functionalized CNTs. For both samples, MWNTs were coated by a layer of organic species whose thickness depends on the molecular size and the quantity covalently attached onto the surfaces of MWNTs (Figure 9). The diameter of MWNT-COOH is about $13-16 \mathrm{~nm}$, while the diameter of $\mathrm{PcH}_{2}-\mathrm{MWNTs}$ is about $17-20 \mathrm{~nm}$. These findings suggest that the average thickness of the $\mathrm{PcH}_{2}$ outside the wellregulated carbon layers is about $2 \mathrm{~nm}$, being greatly in agreement with the size of tert-butyl phthalocyanine.

The Z-scan method has been used to investigate the thirdorder nonlinear optical (NLO) processes, including nonlinear absorption, scattering, and refraction, of functional materials. ${ }^{7,9 a, 10}$ Figure 10 gives the open aperture Z-scan curves of $\mathrm{PcH}_{2-}$ MWNTs for different input fluences with normalized transmission plotted as a function of sample position $z$. All Z-scans exhibited a reduction in the transmission about the focus of the lens, a typical optical limiting characteristic. The depth of reduction changes along with the variations of the on-focus intensity. The minimal normalized transmittance reaches $83 \%$ for a $0.5 \mathrm{mg} / \mathrm{mL}$ solution at the on-focus intensity of $0.17 \mathrm{GW} /$ $\mathrm{cm}^{2}\left(1.02 \mathrm{~J} / \mathrm{cm}^{2}\right)$, which further decreases to $68 \%$ as the intensity increases to $0.61 \mathrm{GW} / \mathrm{cm}^{2}\left(3.66 \mathrm{~J} / \mathrm{cm}^{2}\right)$.

Figure 11 shows the normalized transmission and the scattered signal as functions of incident energy density for $\mathrm{PcH}_{2}$-MWNTs
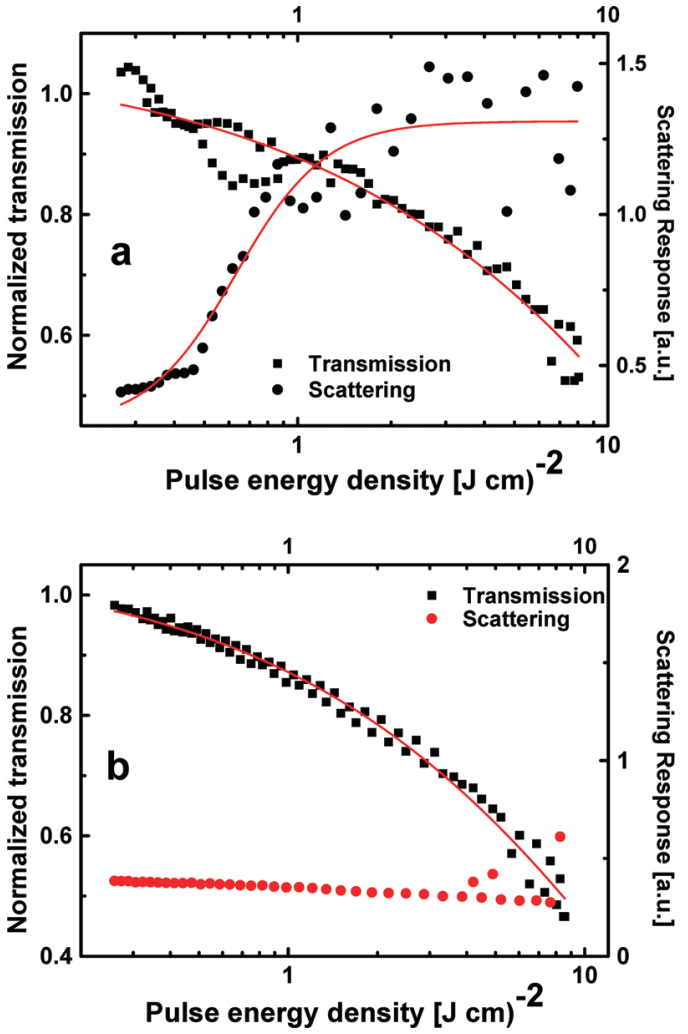

Figure 11. Plots of normalized transmission and scattering response against incident pulse energy density for (a) $\mathrm{PcH}_{2}-\mathrm{MWNTs}$ and (b) $t \mathrm{Bu}_{3} \mathrm{NH}_{2} \mathrm{PcH}_{2}$ in toluene. The solid lines are intended as a visual guide.

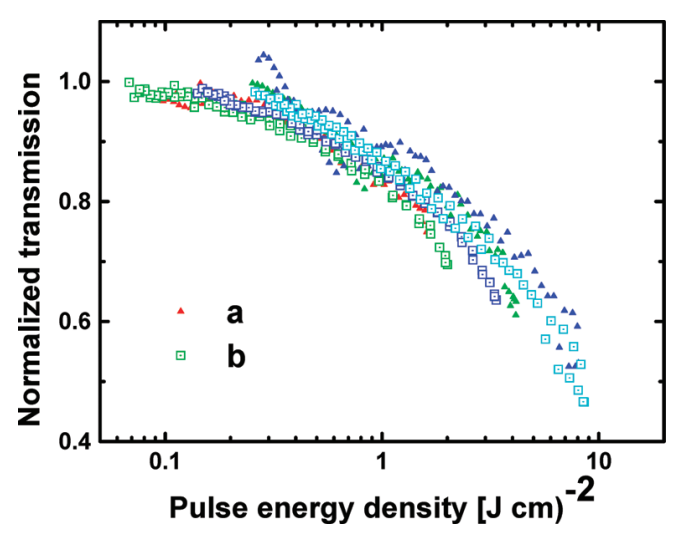

Figure 12. Plot of normalized transmission against incident pulse energy density for (a) $\mathrm{PcH}_{2}-\mathrm{MWNT}$ and (b) $t \mathrm{Bu}_{3} \mathrm{NH}_{2} \mathrm{PcH}_{2}$.

and its RSA counterpart $t \mathrm{Bu}_{3} \mathrm{NH}_{2} \mathrm{PcH}_{2}$. The decreased transmission along with the increase of incident energy in these two compounds implies apparently the potential application for optical limiting. And more, very different scattering behavior was detected from these two sample solutions (Figure 12). $\mathrm{PcH}_{2}$ MWNTs exhibit strong scattering at higher intensities, which evidently comes from MWNT counterpart. In such a nonlinear scattering process, the light absorption from the intense laser pulses induces a very high temperature rising in the nanotubes, which enables the vaporization and ionization of MWNTs to rapidly expand microplasmas. The light is strongly scattered by the microplasmas, leading to the abrupt decrease of the output energy. In addition, the heat is transferred from the MWNTs to surrounding liquid to produce microbubbles, which subsequently scatter light. ${ }^{26}$ In contrast, we did not observe any strong scattering from the pristine $t \mathrm{Bu}_{3} \mathrm{NH}_{2} \mathrm{PcH}_{2}$ solution (see Figure 
TABLE 1: Linear and Nonlinear Optical Coefficients for $t \mathrm{Bu}_{3} \mathrm{NH}_{2} \mathrm{PcH}_{2}$ and $\mathrm{PcH}_{2}$-MWNTs

\begin{tabular}{lccccc}
\hline \multicolumn{1}{c}{ samples } & $\begin{array}{c}\text { conc } \\
{[\mathrm{mg} / \mathrm{mL}]}\end{array}$ & $\begin{array}{c}T \\
{[\%]}\end{array}$ & $\begin{array}{c}\mathrm{Abs} \\
{\left[\mathrm{cm}^{-1}\right]}\end{array}$ & $\begin{array}{c}\beta_{\text {eff }} \\
{[\mathrm{cm} / \mathrm{GW}]}\end{array}$ & $\begin{array}{c}\operatorname{Im}\left\{\chi^{(3)}\right\} \\
{\left[\times 10^{-11} \mathrm{esu}\right]}\end{array}$ \\
\hline $\mathrm{PcH}_{2}-\mathrm{MWNTs}$ & 0.5 & 91 & 0.95 & $39.0 \pm 9.6$ & $1.47 \pm 0.36$ \\
$t \mathrm{Bu}_{3} \mathrm{NH}_{2} \mathrm{PcH}_{2}$ & 0.5 & 89 & 1.18 & $40.1 \pm 6.3$ & $1.51 \pm 0.24$
\end{tabular}

12b), implying that the optical limiting response totally originates from RSA. The slight scattering signal in Figure $12 \mathrm{~b}$ is due to the plasmarization of $t \mathrm{Bu}_{3} \mathrm{NH}_{2} \mathrm{PcH}_{2}$ molecule under higher intensity irradiations. The linear and NLO coefficients of $\mathrm{PcH}_{2}-\mathrm{MWNT}$ and $t \mathrm{Bu}_{3} \mathrm{NH}_{2} \mathrm{PcH}_{2}$ are collected in Table 1 .

Figure 12 compares the optical limiting performances of $t \mathrm{Bu}_{3} \mathrm{NH}_{2} \mathrm{PcH}_{2}$ and $\mathrm{PcH}_{2}-\mathrm{MWNT}$. These two materials show similar linear absorption, nonlinear extinction, and optical limiting properties. It shoud be noted that the nonlinear response of $t \mathrm{Bu}_{3} \mathrm{NH}_{2} \mathrm{PcH}_{2}$ is due to RSA, while that of $\mathrm{PcH}_{2}$-MWNTs is due to both RSA and nonlinear scattering. At the same mass concentration, the contribution of RSA to optical limiting in the former is comparable to that of RSA and scattering in the latter. The RSA and nonlinear scattering could be two conflicted mechanisms for optical limiting, ${ }^{27}$ which may also suppress the whole nonlinear response of $\mathrm{PcH}_{2}$-MWNTs.

\section{Conclusions}

Dissolving CNTs in water and organic solvents, however, is still challenging. To address this problem, one strategy for this issue has been to obtain soluble organic and/or polymeric derivatives through chemical reactions. We designed and prepared a new unsymmetrically substituted metal-free phthalocyanine covalently grafted multiwalled carbon nanotube derivative, $\mathrm{PcH}_{2}-\mathrm{MWNTs}$, through the amidation reaction. The wt $\%$ of MWNTs in the resulting product was found to be $35 \%$ from which one can understand the reason why the resulting material is soluble in some common organic solvents. In contrast to $t \mathrm{Bu}_{3} \mathrm{NH}_{2} \mathrm{PcH}_{2}$, the peak maxima of the Q- and B-bands of $\mathrm{PcH}_{2}$-MWNTs move to the red by $\Delta \lambda=3 \mathrm{~nm}$ and to the blue by $\Delta \lambda=2 \mathrm{~nm}$, respectively, as a consequence of the electronic interactions with MWNTs. A quenching of the singlet excited $\mathrm{PcH}_{2}$ by the covalently linked MWNTs has also been observed. This material exhibits strong nonlinear scattering at higher intensities, which evidently comes from the MWNT counterpart. Future work will be focused on in-depth studies of solution and solid state systems to provide a detailed understanding of the factors affecting the optical limiting response.

Acknowledgment. We are grateful for the financial support of National Natural Science Foundation of China (20676034, 20876046), New Century Excellent Talents in University (NCET050413), Ministry of Education of China (309013), and Shanghai Municipal Educational Commission for the Shuguang fellowship (08GG10) and Shanghai Eastern Schlorship, respectively. J. W. thanks the Science Foundation Ireland (SFI) for his postdoctoral research fellowship. This work was partially supported by the SFI under Grant No. 08/CE/I1432.

\section{References and Notes}

(1) ABC News reported on Dec. 30, 2004. See http://abcnews.go.com. For more information, please visit USAF Institute for National Security Studies (INSS) home page at www. usafa.af.mil/inss.
(2) Spangler, C. W. J. Mater. Chem. 1999, 9, 2013.

(3) (a) Brusatin, G.; Signorini, R. J. Mater. Chem. 2002, 12, 1964. (b) Sun, Y. P.; Riggs, J. E. Int. Rev. Phys. Chem. 1999, 18, 43. (c) De La Torre, G.; Vazquez, P.; Agullo-Lopez, F.; Torres, T. J. Mater. Chem. 1998, $8,1671$.

(4) Nalwa, H. S.; Shirk, J. S. In Phthalocyanines: Properties and Applications; Leznoff, C. C., Lever, A. B. P., Eds.; VCH Publishers, Inc.: New York, 1996; Vol. 4, p 83.

(5) De La Torre, G.; Vazquez, P.; Agullo-Lopez, F.; Torres, T. Chem. Rev. 2004, 104, 3723.

(6) Chen, Y.; Hanack, M.; Araki, Y.; Ito, O. Chem. Soc. Rev. 2005, 34, 517.

(7) O'Flaherty, S. M.; Hold, S. V.; Cook, M. J.; Torres, T.; Chen, Y.; Hanack, M.; Blau, W. J. Adv. Mater. 2003, 15, 19.

(8) Calvete, M.; Yang, G. Y.; Hanack, M. Synth. Met. 2004, 141, 231.

(9) (a) Chen, Y.; EI-Khouly, M. E.; Doyle, J. J.; Lin, Y.; Liu, Y.; Notaras, E.; Blau, W. J.; O'Flaherty, S. M. In Handbook of Organic Electronics and Photonics; American Scientific Publishers: Stevenson Ranch, CA, 2008, 2, 151; (b) Chen, Y.; Hanack, M.; Blau, W. J.; Dini, D.; Doyle, J.; Liu, Y.; Lin, Y.; Bai, J. J. Mater. Sci. 2006, 41, 2169.

(10) For example, (a) Chen, Y.; Barthel, M.; Seiler, M.; Subramanian, L. R.; Bertagnolli, H.; Hanack, M. Angew Chem., Int. Ed. 2002, 41, 3239. (b) Bertagnolli, H.; Blau, W. J.; Chen, Y.; Dini, D.; Feth, M. P.; O'Flaherty, S. M.; Hanack, M.; Krishnan, V. J. Mater. Chem. 2005, 15, 683. (c) Chen, Y.; Fujitsuka, M.; O’Flaherty, S. M.; Hanack, M.; Ito, O.; Blau, W. J. Adv. Mater. 2003, 15, 899. (d) Chen, Y.; EI-Khouly, M. E.; Sasaki, M.; Araki, Y.; Ito, O. Org. Lett. 2005, 7, 1613. (e) Chen, Y.; O'Flaherty, S. M.; Fujitsuka, M.; Hanack, M.; Subramanian, L. R.; Ito, O.; Blau, W. J. Chem. Mater. 2002, 14, 5163. (f) Yang, G. Y.; Hanack, M.; Lee, Y. W.; Chen, Y.; Yuen, M. L. K.; Vagin, S.; Dini, D. Chem.-Eur. J. 2003, 9, 2758. (g) Chen, Y.; Dini, D.; Hanack, M.; Fujitsuka, M.; Ito, O. Chem. Commun. 2004, 340. (h) Chen, Y.; He, N.; Doyle, J. J.; Liu, Y.; Zhuang, X.; Blau, W. J. J. Photochem. Photobiol., A 2007, 189, 414.

(11) Chen, Y.; Lin, Y.; Liu, Y.; Doyle, J.; He, N.; Zhuang, X.; Bai, J.; Blau, W. J. J. Nanosci. Nanotechnol. 2007, 7, 1268.

(12) Tasis, D.; Tagmatarchis, N.; Bianco, A.; Prato, M. Chem. Rev. 2006, 106,1105 765.

(13) de la Torre, G.; Blau, W.; Torres, T. Nanotechnology 2003, 14,

(14) Ballesteros, B.; de la Torre, G.; Ehli, X.; Rahman, G. M. A.; AgulloRueda, F.; Guld, D. M.; Torres, T. J. Am. Chem. Soc. 2007, 129, 5061.

(15) Kudrevich, S. V.; Ali, H.; van Lier, J. E. J. Chem. Soc., Perkin Trans. 1 1994, 2767.

(16) (a) Xu, Y.; Cao, C.; Kong, H.; Yan, D.; Jin, Y. Z.; Watts, P. C. P. Macromolecules 2004, 37, 8846. (b) Kong, H.; Cao, C.; Yin, D. J. Am. Chem. Soc. 2004, 126, 412.

(17) (a) Hamon, M. A.; Chen, J.; Hu, H.; Chen, Y.; Itkis, M. E.; Rao, A. M.; Eklund, P. C.; Haddon, R. C. Adv. Mater. 1999, 11, 834. (b) Chen, J.; Hamon, M. A.; Hu, H.; Chen, Y.; Rao, A. M.; Eklund, P. C.; Haddon, R. C. Science 1998, 282, 95.

(18) Chen, G. Z.; Thiel, W.; Hirsch, A. ChemPhysChem. 2003, 93.

(19) (a) Gao, C.; Jin, Y. Z.; Kong, H.; Whitby, R. L. D.; Acquah, S. F. A.; Chen, G. Y.; Qian, H.; Hartschuh, A.; Silva, S. R. P.; Henley, S.; Fearon, P.; Kroto, H. W.; Walton, D. R. M. J. Phys. Chem. B 2005, 109, 11925. (b) Guo, Z.; Du, F.; Ren, D.; Chen, Y.; Zheng, J.; Liu, Z.; Tian, J. J. Mater. Chem. 2006, 16, 3021. (c) Bahr, J. L.; Tour, J. M. J. Mater. Chem. 2002, 12, 1952.

(20) Dresselhaus, M. S.; Dresselhaus, G.; Saito, R.; Jorio, A. Phys. Rep. 2005, 409, 47.

(21) Cioffi, C.; Campidelli, S.; Sooambar, C.; Marcaccio, M.; Marcolongo, G.; Meneghetti, M.; Paolucci, D.; Paolucci, F.; Ehli, C.; Rahman, G. M. A.; Sgobba, V.; Guldi, D. M.; Prato, M. J. Am. Chem. Soc. 2007, $129,3938$.

(22) (a) Liu, Z. B.; Tian, J. G.; Guo, Z.; Ren, D. M.; Du, F.; Zheng, J. Y.; Chen, Y. S. Adv. Mater. 2008, 20, 511. (b) Guo, Z.; Du, F.; Ren, D.; Chen, Y.; Zheng, J.; Liu, Z.; Tian, J. J. Mater. Chem. 2006, 16, 3021.

(23) Chen, Y.; Huang, Z. E.; Cai, R. F. J. Polym. Sci., Part B: Polym. Phys. 1996, 34, 631.

(24) Snow, A. W.; Shirk, J. S.; Peebles, L. H., Jr. Abstracts of the First International Conference on Porphyrins and Phthalocyanines (ICPP-1), Dijon, France, June 25-30, 2000; p 177.

(25) Lin, S. T.; Wei, K. L.; Lee, T. M.; Chiou, K. C.; Lin, J. J. Nanotechnology 2006, 17, 3197.

(26) (a) Wang, J.; Blau, W. J. Appl. Phys. B: Lasers Opt. 2008, 91, 521. (b) Wang, J.; Blau, W. J. J. Phys. Chem. C 2008, 112, 2298.

(27) Wang, J.; Blau, W. J. Chem. Phys. Lett. 2008, 465, 265.

JP9006813 